\title{
THE NUMBER OF PRIME FACTORS OF INTEGERS WITH DENSE DIVISORS
}

\author{
ANDREAS WEINGARTNER
}

\begin{abstract}
We show that for integers $n$, whose ratios of consecutive divisors are bounded above by an arbitrary constant, the normal order of the number of prime factors is $C \log \log n$, where $C=\left(1-e^{-\gamma}\right)^{-1}=$ $2.280 \ldots$ and $\gamma$ is Euler's constant. We explore several applications and resolve a conjecture of Margenstern about practical numbers.
\end{abstract}

\section{INTRODUCTION}

We say that a positive integer $n$ is $t$-dense if the ratios of consecutive divisors of $n$ do not exceed $t$. Let $\mathcal{D}(x, t)$ denote the set of $t$-dense integers $n \leq x$ and write $D(x, t)=|\mathcal{D}(x, t)|$. Let $\omega(n)$ (resp. $\Omega(n))$ be the number of prime factors of $n$, counted without (resp. with) multiplicity.

Theorem 1 gives the average and normal order of $\omega(n)$ and $\Omega(n)$ for the $t$-dense integers. We write $\log _{2} x$ for $\log \log x$ and define $E(x, t)$, the approximate expected value of $\omega(n)$ on $\mathcal{D}(x, t)$, by

$$
E(x, t):=C \log _{2} x-(C-1) \log _{2} t, \quad C:=\left(1-e^{-\gamma}\right)^{-1}=2.280291 \ldots
$$

Theorem 1. Let $\xi(x) \rightarrow \infty$. Uniformly for $x \geq t \geq 2$,

$$
\frac{\sum_{n \in \mathcal{D}(x, t)} \omega(n)}{D(x, t)}=E(x, t)+O(1)
$$

and

$$
\left|\left\{n \in \mathcal{D}(x, t):|\omega(n)-E(x, t)|>\xi(x) \sqrt{\log _{2} x}\right\}\right| \ll \frac{D(x, t)}{\xi(x)^{2}} .
$$

These results also hold with $\Omega$ in place of $\omega$.

Note that $\log _{2} x \leq E(x, t) \leq C \log _{2} x+O(1)$, as $x \geq t \geq 2$. If $t=x$, then $E(x, t)=\log _{2} x$ and $\mathcal{D}(x, t)=[1, x] \cap \mathbb{N}$, so Theorem 1 contains the well-known results about the average and normal order of $\omega(n)$ on $\mathbb{N}$. If $t \geq 2$ is constant, then $E(x, t)=C \log _{2} x+O(1)$, so that the average and normal order of $\omega(n)$ for $t$-dense integers $n$ is $C \log _{2} n$.

The $t$-dense integers are a special case of a family of integer sequences that arise as follows. Let $\theta$ be an arithmetic function. Let $\mathcal{B}=\mathcal{B}_{\theta}$ be the

Date: November 11, 2021.

2010 Mathematics Subject Classification. 11N25, 11N37. 
set of positive integers containing $n=1$ and all those $n \geq 2$ with prime factorization $n=p_{1}^{\alpha_{1}} \cdots p_{k}^{\alpha_{k}}, p_{1}<p_{2}<\ldots<p_{k}$, which satisfy

$$
p_{i} \leq \theta\left(p_{1}^{\alpha_{1}} \cdots p_{i-1}^{\alpha_{i-1}}\right) \quad(1 \leq i \leq k) .
$$

We write $\mathcal{B}(x)=\mathcal{B} \cap[1, x]$ and $B(x)=|\mathcal{B}(x)|$. When $\theta(n)=n t$, then $\mathcal{B}$ is the set of $t$-dense integers $[6,10,14]$. If $\theta(n)=\sigma(n)+1$, where $\sigma(n)$ is the sum of the positive divisors of $n$, then $\mathcal{B}$ is the set of practical numbers $[2,6,7,8,9,10,14]$, i.e. integers $n$ such that every $m \leq n$ can be expressed as a sum of distinct positive divisors of $n$.

We will derive Corollaries 1 through 3 from Theorem 1 in Section 2.

Corollary 1. Assume $\theta$ satisfies $\max (2, n) \leq \theta(n) \leq n f(n)$, where $f$ is non-decreasing. Let $\xi(x) \rightarrow \infty$. We have

$$
\frac{\sum_{n \in \mathcal{B}(x)} \omega(n)}{B(x)}=C \log _{2} x\left\{1+O\left(\left(\frac{\log f(x)}{\log _{2} x}\right)^{1 / 3}\right)\right\}
$$

and

$$
\left|\left\{n \in \mathcal{B}(x):\left|\omega(n)-C \log _{2} x\right|>\xi(x) \sqrt{\log _{2} x}\right\}\right| \ll B(x) \frac{\log f(x)}{\xi(x)^{2}} .
$$

These results also hold with $\Omega$ in place of $\omega$.

Conjecture 5 of Margenstern [2] proposes that, for practical numbers, $\sum_{n \in \mathcal{B}(x)} \omega(n) \sim \mu x /(\log x)^{\eta}$, for some constants $\mu>0$ and $1 / 2<\eta<1$. The estimate (4) disproves this conjecture, since $B(x) \sim c_{\theta} x / \log x$ by [14, Thm. 1.2], if $\max (2, n) \leq \theta(n) \ll n(\log 2 n) /\left(\log _{2} 3 n\right)^{1+\varepsilon}$ for $n \geq 1$.

Corollary 2 shows that almost all large practical numbers $n$ have about $C \log _{2} n$ prime factors.

Corollary 2. If $\theta$ satisfies $\max (2, n) \leq \theta(n) \ll n(\log 2 n)^{o(1)}$, then the average and normal order of $\omega(n)$ on $\mathcal{B}$ is $C \log _{2} n$. That is, as $x \rightarrow \infty$,

$$
\sum_{n \in \mathcal{B}(x)} \omega(n) \sim \sum_{n \in \mathcal{B}(x)} C \log _{2} n
$$

and all but $o(B(x))$ integers $n \in \mathcal{B}(x)$ satisfy

$$
\omega(n)=(1+o(1)) C \log _{2} n .
$$

These results also hold with $\Omega$ in place of $\omega$.

Let $\tau(n)$ be the number of positive divisors of $n$.

Corollary 3. If $\theta$ satisfies $\max (2, n) \leq \theta(n) \ll n(\log 2 n)^{o(1)}$, then

$$
(\log x)^{C \log 2-o(1)}<\frac{\sum_{n \in \mathcal{B}(x)} \tau(n)}{B(x)} \ll(\log x)^{e \log 2},
$$

as $x \rightarrow \infty$, and all but $o(B(x))$ integers $n \in \mathcal{B}(x)$ satisfy

$$
\tau(n)=(\log n)^{C \log 2+o(1)}=(\log n)^{1.580577 \ldots+o(1)} .
$$


Conjecture 4 of Margenstern [2] says that, in the case of practical numbers, $\sum_{n \in \mathcal{B}(x)} \tau(n) \sim \nu x(\log x)^{\delta}$, for constants $1 / 2<\nu, \delta<1$. The estimate (8) implies $\delta \in[C \log 2-1, e \log 2-1]=[0.580 \ldots, 0.884 \ldots]$, since $B(x) \asymp x / \log x$. In [17], we prove this conjecture with $\delta=0.713 \ldots$ and some constant $\nu>0$.

The next two corollaries are improvements to the lower bounds of Theorems 1 and 3 of [5]. The proofs of both of these theorems rely on the fact that almost all $n \in \mathcal{B}$ satisfy $\Omega(n)<(e+\varepsilon) \log _{2} n$. With Corollary 2 , this can be improved to $\Omega(n)<(C+\varepsilon) \log _{2} n$, under the assumption $\theta(n) \ll n(\log n)^{o(1)}$. In the lower bound of [5, Thm. 1] for the count of practical numbers that are also shifted primes, which has an exponent of $(e+1) \log (e+1)-e \log (e)+1+\varepsilon=3.16470 \ldots$, we can replace $e$ by $C$ to get $3.01711 \ldots$.

Corollary 4. Fix a nonzero integer $h$ and assume $\theta$ satisfies

$$
\max (2, n) \leq \theta(n) \ll n(\log 2 n)^{o(1)}, \quad \theta(m n) \ll m^{O(1)} \theta(n) \quad(n, m \in \mathbb{N}) .
$$

We have

$$
\frac{x}{(\log x)^{3.01712}} \ll_{h} \mid\{p \leq x: p \text { prime }, p-h \in \mathcal{B}\} \mid \ll_{h} \frac{x}{(\log x)^{2}},
$$

where $h$ is not divisible by $\prod_{p \leq \theta(1)} p$ in the lower bound.

Similarly, in the lower bound of [5, Thm. 3] for the count of twin practical numbers, which has an exponent of $2+4 e \log 2+\varepsilon=9.53667 \ldots$, we can replace $e$ by $C$ to get $8.32230 \ldots$

Corollary 5. Fix a nonzero integer $h$ and assume $\theta$ satisfies (10). We have

$$
\frac{x}{(\log x)^{8.32231}} \ll_{h}|\{n \leq x: n \in \mathcal{B}, n+h \in \mathcal{B}\}| \ll_{h} \frac{x}{(\log x)^{2}} .
$$

For the lower bound, assume that (i) $n \in \mathcal{B}$ and $m \leq 3 n /|h|$ imply $m n \in \mathcal{B}$, and (ii) if $\theta(1)<3$, then $h \in 2 \mathbb{Z}$ if $\theta(2) \geq 3$, and $h \in 4 \mathbb{Z}$ if $\theta(2)<3$.

Conditions (i) and (ii) in Corollary 5 are satisfied by the practical numbers and by the 2-dense integers for any nonzero even integer $h$, and by the $t$ dense integers for any nonzero integer $h$, provided $t \geq 3$.

The $\varphi$-practical numbers $[4,12]$ are integers $n$ such that $x^{n}-1$ has a divisor in $\mathbb{Z}[x]$ of every degree up to $n$. Although not an example of a set $\mathcal{B}_{\theta}$, they are a superset of $\mathcal{B}_{\theta_{1}}$ with $\theta_{1}(n)=n+1$, and a subset of $\mathcal{B}_{\theta_{2}}$ with $\theta_{2}(n)=n+2$. Therefore, Corollaries 1 through 5 also apply to the $\varphi$-practical numbers, provided $h$ is odd in the lower bound of Corollary 4 , while $h$ is even in the lower bound of Corollary 5 .

Theorem 1 is a consequence of Theorems 2 and 3 . Theorem 2 gives an estimate for

$$
D_{q}(x)=D_{q}(x, t):=|\{n \in \mathcal{D}(x, t): q \mid n\}|,
$$

when $q$ has a bounded number of prime factors. As in the case $q=1$ (see [14, Thm. 1.3]), the main term contains the function $d(v)$, which is defined 
by $d(v)=0$ for $v<0$ and

$$
d(v)=1-\int_{0}^{\frac{v-1}{2}} \frac{d(u)}{u+1} w\left(\frac{v-u}{u+1}\right) d u \quad(v \geq 0),
$$

where $w(u)$ denotes Buchstab's function.

Theorem 2. Let $k \in \mathbb{N} \cup\{0\}$ be fixed. Uniformly for $x \geq 1, t \geq 2, q \in \mathbb{N}$ with $\Omega(q)=k, v=\log x / \log t$, we have

$$
\begin{aligned}
& D_{q}(x, t)=x d(v) \eta_{q, t}\left\{1+O_{k}\left(\frac{1}{\log x t}+\frac{\log 2 q \log q t}{\log ^{2} x t}\right)\right\}+O(1), \\
& D_{q}(x, t)=x d(v) \eta_{q, t}\left\{1+O_{k}\left(\frac{\log 2 q}{\log 2 x}\right)\right\}
\end{aligned}
$$

where

$$
q^{-1} \ll \eta_{q, t} \ll_{k} q^{-1} .
$$

Corollary 6. Let $k \in \mathbb{N} \cup\{0\}$ be fixed. Uniformly for $x \geq 1, t \geq 2, q \in \mathbb{N}$ with $\Omega(q)=k$, we have

$$
D_{q}(x, t)=\frac{c_{q} x}{\log x t}\left\{1+O_{k}\left(\frac{1}{\log x t}+\frac{\log ^{2} q t}{\log ^{2} x t}\right)\right\}+O(1),
$$

where

$$
c_{q}=c_{q, t}=C \eta_{q, t} \log t, \quad q^{-1} \log t \ll c_{q} \ll_{k} q^{-1} \log t,
$$

and

$$
D_{q}(x, t) \ll_{k} \frac{x \log t}{q \log x t} .
$$

The estimates (15) and (16) follow from (12) and (14), since $d(v)=$ $C(v+1)^{-1}\left\{1+O\left((v+1)^{-2}\right)\right\}$ by [13, Thm. 1]. The upper bound (17) follows from (13), because $D_{q}(x, t)=0$ if $q>x$.

Theorem 3 gives estimates for $c_{q}$ when $q$ is a prime or a product of two primes. These estimates are needed to derive Theorem 1 from Theorem 2.

Theorem 3. Let $p \leq q$ be primes. The constant factor in (15) satisfies

$$
\begin{gathered}
c_{\theta}:=c_{1}=C(\log t-\gamma)+O\left(e^{-\sqrt{\log t}}\right) \\
q c_{q}=C c_{\theta}\left\{1+O\left(\frac{1}{\log q}+\frac{\log ^{2} t}{\log ^{2} q}\right)\right\} \\
q c_{q}=c_{\theta}+C \log q+O(\exp (-\sqrt{\log t})) \quad(q \leq t) \\
p q c_{p q}=C^{2} c_{\theta}\left\{1+O\left(\frac{1}{\log p}+\frac{\log ^{2} t}{\log ^{2} p}+\frac{\log ^{2} p}{\log ^{2} q}\right)\right\} \\
p q c_{p q}=\left(C c_{\theta}+C^{2} \log p\right)\left\{1+O\left(\frac{1}{\log q}+\frac{\log ^{2} t}{\log ^{2} q}+e^{-\sqrt{\log t}}\right)\right\} \quad(p \leq t) \\
p q c_{p q}=c_{\theta}+C \log p q+O\left(e^{-\sqrt{\log t}}\right) \quad(p \leq q \leq t)
\end{gathered}
$$


In Section 2 we derive Corollaries 1, 2 and 3 from Theorem 1. Section 3 contains several lemmas, used in the proofs of Theorems 2 and 3 , about members of $\mathcal{B}$ that are multiples of a natural number $q$. The proof of Theorem 2 is given in Section 4. In Section 5 we establish Theorem 3 with the help of Corollary 6, which is a consequence of Theorem 2. Finally, in Section 6 we apply Theorems 2 and 3 to prove Theorem 1.

\section{Proof of Corollaries 1,2 And 3}

Lemma 1. We have

$$
\begin{gathered}
D(x, t) \ll \frac{x \log t}{\log x t} \quad(x>1 / t, t \geq 2), \\
D(x, t) \gg \frac{x \log t}{\log x t} \quad(x \geq 1, t \geq 2), \\
D(x / q, t)-D(q / t, t) \leq D_{q}(x, t) \leq D(x / q, q t) \quad(x \geq 0, t \geq 2, q \geq 1) .
\end{gathered}
$$

Proof. The first two estimates follow from [6, Thm. 1].

If $m \in \mathcal{D}(x / q, t)$ and $m>q / t$, then $m q \in \mathcal{D}_{q}(x, t)$. This shows that $D(x / q, t)-D(q / t, t) \leq D_{q}(x, t)$.

If $n \in \mathcal{D}(x, t)$ and $q \mid n$, we write $n=q m$ and observe that $m \in \mathcal{D}(x / q, q t)$. Thus, $D_{q}(x, t) \leq D(x / q, q t)$.

Proof of Corollary 1. We first show (5). For $1 \leq n \leq x$, we have $\max (2, n) \leq$ $\theta(n) \leq n f(n) \leq n f(x)$. Thus, $\mathcal{B}(x) \subset \mathcal{D}(x, f(x))$. If $f(x) \leq x,(2)$ yields

$$
\left|\left\{n \in \mathcal{B}(x):|\omega(n)-E(x, f(x))|>\frac{\xi(x)}{2} \sqrt{\log _{2} x}\right\}\right| \ll \frac{D(x, f(x))}{\xi(x)^{2}} .
$$

The assumption $\theta(n) \geq \max (2, n)$ implies $B(x) \gg x / \log x$, by [14, Thm. 1.2]. By Lemma 1,

$$
\frac{D(x, f(x))}{\xi(x)^{2}} \ll \frac{x \log f(x)}{\xi(x)^{2} \log x} \ll B(x) \frac{\log f(x)}{\xi(x)^{2}} .
$$

The result being trivial if $\log f(x)>\xi(x)^{2}$, we may assume $\log f(x) \leq \xi(x)^{2}$, so that

$$
\left|E(x, f(x))-C \log _{2} x\right|=\left|(C-1) \log _{2} f(x)\right| \leq \frac{\xi(x)}{2} \sqrt{\log _{2} x},
$$

for $x \geq x_{0}$. Thus, (5) holds if $f(x) \leq x$. If $f(x)>x$, then $\xi(x)^{2} \geq \log f(x)>$ $\log x$, so that $\left|\omega(n)-C \log _{2} x\right|>\xi(x) \sqrt{\log _{2} x}$ implies $\omega(n)>\xi(x)>\sqrt{\log x}$. The result now follows from Nicolas' Theorem [3], an asymptotic estimate for the quantity $|\{n \leq x: \Omega(n)=k\}|$, which easily implies

$$
\left|\left\{n \leq x: \Omega(n) \geq y \log _{2} x\right\}\right| \ll \frac{x}{(\log x)^{y \log 2-1}},
$$

uniformly for $x \geq 2$ and $y \geq 2+\delta$, for any fixed $\delta>0$. 
Next, we show that (5) implies (4). From (24) we have

$$
\left|\left\{n \leq x: \omega(n) \geq 6 \log _{2} x\right\}\right| \leq\left|\left\{n \leq x: \Omega(n) \geq 6 \log _{2} x\right\}\right| \ll \frac{B(x)}{\log ^{2} x} .
$$

Since $\omega(n) \leq \Omega(n) \ll \log n$, the contribution to $\sum_{n \in \mathcal{B}(x)} \omega(n)$ from $n$ with $\omega(n)>6 \log _{2} x$ is $\ll B(x) / \log x$, while the contribution from $n$ with $\omega(n) \leq$ $6 \log _{2} x$ and $\left|\omega(n)-C \log _{2} x\right|>\xi(x) \sqrt{\log _{2} x}$ is

$$
\ll\left(6 \log _{2} x\right) B(x) \frac{\log f(x)}{\xi(x)^{2}},
$$

by (5). The contribution to $\sum_{n \in \mathcal{B}(x)} \omega(n)$ from $n$ with $\omega(n) \leq 6 \log _{2} x$ and $\left|\omega(n)-C \log _{2} x\right| \leq \xi(x) \sqrt{\log _{2} x}$ is

$$
B(x)\left(1+O\left(\frac{\log f(x)}{\xi(x)^{2}}+\frac{1}{\log ^{2} x}\right)\right) C \log _{2} x\left(1+O\left(\frac{\xi(x)}{\sqrt{\log _{2} x}}\right)\right) .
$$

If $\log f(x) \leq \log _{2} x$, (4) now follows with $\xi(x)=(\log f(x))^{1 / 3}\left(\log _{2} x\right)^{1 / 6}$. If $\log f(x)>\log _{2} x$, (4) follows directly from (25). The argument works the same with $\Omega(n)$ in place of $\omega(n)$.

Proof of Corollary 2. Assume $\max (2, n) \leq \theta(n) \ll n(\log 2 n)^{o(1)}$. Define $f(x)=\max _{n \leq x} \theta(n) / n$, so that $f$ is non-decreasing and $f(x)=(\log x)^{o(1)}$ as $x \rightarrow \infty$, that is $\log f(x)=o\left(\log _{2} x\right)$. The relation (6) follows from (4). Choosing $\xi(x)=\left(\log f(x) \log _{2} x\right)^{1 / 4}$ in (5) yields (7).

Lemma 2. Let $\varepsilon>0$. For $2 \leq \alpha \leq 4-\varepsilon$ we have

$$
\sum_{\substack{n \leq x \\ \Omega(n) \geq \alpha \log _{2} x}} \tau(n) \ll x(\log x)^{\alpha(\log 2-\log \alpha+1)-1} .
$$

Proof. This is a variation of Exercise 05 in [1]. Write $y^{\Omega(n)}=\sum_{d \mid n} f(d)$, so that $f(n)$ is multiplicative and $f\left(p^{k}\right)=y^{k}(1-1 / y)$ for $k \geq 1$, by Möbius inversion. For $0 \leq y \leq 2-\varepsilon$,

$$
\begin{aligned}
\sum_{n \leq x} \tau(n) y^{\Omega(n)} & =\sum_{n \leq x} \tau(n) \sum_{d \mid n} f(d) \leq \sum_{d \leq x} f(d) \tau(d) \sum_{m \leq x / d} \tau(m) \\
& \leq x \log x \sum_{d \leq x} f(d) \tau(d) / d \leq x \log x \sum_{P^{+}(d) \leq x} f(d) \tau(d) / d \\
& =x \log x \prod_{p \leq x} \sum_{k \geq 0} f\left(p^{k}\right) \tau\left(p^{k}\right) / p^{k} \ll x(\log x)^{2 y-1} .
\end{aligned}
$$

If $1 \leq y \leq 2-\varepsilon$, we get

$$
\sum_{\substack{n \leq x \\ \Omega(n) \geq \alpha \log _{2} x}} \tau(n) y^{\alpha \log _{2} x} \ll x(\log x)^{2 y-1} .
$$

The result now follows with $y=\alpha / 2$. 
Proof of Corollary 3. Since $2^{\omega(n)} \leq \tau(n) \leq 2^{\Omega(n)}$ for all $n \geq 1$, the estimate (9) and the lower bound in (8) follow at once from (7). For the upper bound in (8), we write

$$
\sum_{\substack{n \in \mathcal{B}(x) \\ \Omega(n) \leq e \log _{2} x}} \tau(n) \leq B(x) 2^{e \log _{2} x}=B(x)(\log x)^{e \log 2}
$$

and

$$
\sum_{\substack{n \in \mathcal{B}(x) \\ \Omega(n) \geq e \log _{2} x}} \tau(n) \leq \sum_{\substack{n \leq x \\ \Omega(n) \geq e \log _{2} x}} \tau(n) \ll x(\log x)^{e \log 2-1} \ll B(x)(\log x)^{e \log 2},
$$

by Lemma 2 with $\alpha=e$.

\section{Multiples of $q$ IN $\mathcal{B}$}

In this section we develop some general identities for sets $\mathcal{B}$, defined by (3), with

$$
\theta: \mathbb{N} \rightarrow \mathbb{R} \cup\{\infty\}, \quad \theta(1) \geq 2, \quad \theta(n) \geq P^{+}(n) \quad(n \geq 2),
$$

where $P^{+}(n)$ denotes the largest prime factor of $n$. Let

$$
\Phi(x, y)=1_{x \geq 1}+\left|\left\{2 \leq n \leq x: P^{-}(n)>y\right\}\right|,
$$

where $P^{-}(n)$ denotes the smallest prime factor of $n$. Let

$$
\psi(n):= \begin{cases}1 & \text { if } n \in \mathcal{B} \\ 0 & \text { else. }\end{cases}
$$

and define

$$
\begin{aligned}
\lambda_{n}(s) & :=\frac{\psi(n)}{n^{s}} \prod_{p \leq \theta(n)}\left(1-\frac{1}{p^{s}}\right), \quad \lambda_{n}:=\lambda_{n}(1), \\
\mu_{n}(s) & :=\sum_{p \leq \theta(n)} \frac{\log p}{p^{s}-1}-\log n, \quad \mu_{n}:=\mu_{n}(1) .
\end{aligned}
$$

Lemma 3. Let $\theta$ satisfy (26) and let $q_{1} \leq q_{2} \leq \ldots \leq q_{k}$ be primes. For $x \geq 0$,

$$
\sum_{n \geq 1} \psi(n) \Phi\left(\frac{x}{n}, \theta(n)\right)=\lfloor x\rfloor
$$

and

$$
\sum_{\substack{n \geq 1 \\ q_{1} \cdots q_{k} \mid n}} \psi(n) \Phi\left(\frac{x}{n}, \theta(n)\right)=\sum_{\substack{\theta(n) \geq q_{k} \\ q_{1} \cdots q_{k-1} \mid n}} \psi(n) \Phi\left(\frac{x}{n q_{k}}, \theta(n)\right) .
$$

Proof. The relation (27) is [15, Lemma 3]. We will show (28). Every $m \in$ $q_{1} \cdots q_{k} \mathbb{N}$ factors uniquely as $m=n r$ where $n \in \mathcal{B}$ and $P^{-}(r)>\theta(n)$ if $r>1$. If $q_{1} \nmid n$ then $\theta(n)<q_{1}$. If $q_{1} \mid n$, let $j$ be the largest index such that 
$q_{1} \cdots q_{j} \mid n$, so that $q_{j+1}>\theta(n)$ if $j<k$. We count all integer multiples of $q_{1} \cdots q_{k}$ up to $x$ according to $j$ and $n$ :

$$
\begin{aligned}
& \left\lfloor\frac{x}{q_{1} \cdots q_{k}}\right\rfloor=\sum_{\theta(n)<q_{1}} \psi(n) \Phi\left(\frac{x}{n q_{1} \cdots q_{k}}, \theta(n)\right) \\
& \quad+\sum_{j=1}^{k-1} \sum_{\substack{\theta(n)<q_{j+1} \\
q_{1} \cdots q_{j} \mid n}} \psi(n) \Phi\left(\frac{x}{n q_{j+1} \cdots q_{k}}, \theta(n)\right)+\sum_{q_{1} \cdots q_{k} \mid n} \psi(n) \Phi\left(\frac{x}{n}, \theta(n)\right) .
\end{aligned}
$$

We can now establish (28) by induction on $k$. When $k=1,(29)$ and (27) yield (28). For the inductive step, we write the inner sum of (29) as

$$
\sum_{\substack{\theta(n)<q_{j+1} \\ q_{1} \cdots q_{j} \mid n}}=\sum_{q_{1} \cdots q_{j} \mid n}-\sum_{\substack{\theta(n) \geq q_{j+1} \\ q_{1} \cdots q_{j} \mid n}}
$$

and use the inductive hypothesis on the sum $\sum_{q_{1} \cdots q_{j} \mid n}$ to get

$$
\begin{gathered}
\sum_{\substack{\theta(n)<q_{j+1} \\
q_{1} \cdots q_{j} \mid n}} \psi(n) \Phi\left(\frac{x}{n q_{j+1} \cdots q_{k}}, \theta(n)\right)=\sum_{\substack{\theta(n) \geq q_{j} \\
q_{1} \cdots q_{j-1} \mid n}} \psi(n) \Phi\left(\frac{x}{n q_{j} \cdots q_{k}}, \theta(n)\right) \\
-\sum_{\substack{\theta(n) \geq q_{j+1} \\
q_{1} \cdots q_{j} \mid n}} \psi(n) \Phi\left(\frac{x}{n q_{j+1} \cdots q_{k}}, \theta(n)\right) .
\end{gathered}
$$

Thus, the sum over $j$ in (29) is telescoping and the result follows from (27).

Lemma 4. Let $\theta$ satisfy (26) and let $q_{1} \leq q_{2} \leq \ldots \leq q_{k}$ be primes. For $\operatorname{Re}(s)>1$ we have

$$
\sum_{n \geq 1} \lambda_{n}(s)=1
$$

and

$$
\sum_{\substack{n \geq 1 \\ q_{1} \cdots q_{k} \mid n}} \lambda_{n}(s)=\frac{1}{q_{k}^{s}} \sum_{\substack{\theta(n) \geq q_{k} \\ q_{1} \cdots q_{k-1} \mid n}} \lambda_{n}(s) .
$$

Both relations hold at $s=1$ if $B(x)=o(x)$.

Proof. The relation (30) is [16, Lemma 1] when $\operatorname{Re}(s)>1$ and $[15$, Theorem 1 ] when $s=1$. The proof of (31) mirrors that of (28). We first assume $\operatorname{Re}(s)>1$. Every $m \in q_{1} \cdots q_{k} \mathbb{N}$ factors uniquely as $m=n r$ where $n \in \mathcal{B}$ and $P^{-}(r)>\theta(n)$ if $r>1$. If $q_{1} \nmid n$ then $\theta(n)<q_{1}$. If $q_{1} \mid n$, let $j$ be the largest index such that $q_{1} \cdots q_{j} \mid n$, so that $q_{j+1}>\theta(n)$ if $j<k$. We rearrange 
the terms of the Dirichlet series $\sum_{q_{1} \cdots q_{k} \mid m} m^{-s}$ according to $n$ and $j$. After dividing by $\zeta(s)$, this shows that, for $\operatorname{Re}(s)>1$,

$$
\frac{1}{\left(q_{1} \cdots q_{k}\right)^{s}}=\sum_{\theta(n)<q_{1}} \frac{\lambda_{n}(s)}{\left(q_{1} \cdots q_{k}\right)^{s}}+\sum_{j=1}^{k-1} \sum_{\substack{\theta(n)<q_{j+1} \\ q_{1} \cdots q_{j} \mid n}} \frac{\lambda_{n}(s)}{\left(q_{j+1} \cdots q_{k}\right)^{s}}+\sum_{q_{1} \cdots q_{k} \mid n} \lambda_{n}(s) .
$$

We establish (31) by induction on $k$. When $k=1$, the result follows from applying (30) to the first sum of (32). For the inductive step, note that the inner sum in $(32)$ is

$$
\begin{aligned}
\sum_{\substack{\theta(n)<q_{j+1} \\
q_{1} \cdots q_{j} \mid n}} \frac{\lambda_{n}(s)}{\left(q_{j+1} \cdots q_{k}\right)^{s}} & =\sum_{q_{1} \cdots q_{j} \mid n} \frac{\lambda_{n}(s)}{\left(q_{j+1} \cdots q_{k}\right)^{s}}-\sum_{\substack{\theta(n) \geq q_{j+1} \\
q_{1} \cdots q_{j} \mid n}} \frac{\lambda_{n}(s)}{\left(q_{j+1} \cdots q_{k}\right)^{s}} \\
& =\sum_{\substack{\theta(n) \geq q_{j}\left|n \\
q_{1} \cdots q_{j-1}\right| n}} \frac{\lambda_{n}(s)}{\left(q_{j} \cdots q_{k}\right)^{s}}-\sum_{\substack{\theta(n) \geq q_{j+1} \\
q_{1} \cdots q_{j} \mid n}} \frac{\lambda_{n}(s)}{\left(q_{j+1} \cdots q_{k}\right)^{s}},
\end{aligned}
$$

by the inductive hypothesis. Thus, the sum over $j$ in (32) is a telescoping sum and the result follows from (30).

If $B(x)=o(x)$, the validity of (31) at $s=1$ follows from (28), in much the same way that the validity of (30) at $s=1$ follows from (27), which is demonstrated in the proof of [15, Thm. 1].

Lemma 5. Let $\theta$ satisfy (26) and let $q_{1} \leq q_{2} \leq \ldots \leq q_{k}$ be primes. For $\operatorname{Re}(s)>1$ we have

$$
\sum_{n \geq 1} \lambda_{n}(s) \mu_{n}(s)=0
$$

and

$$
\sum_{\substack{n \geq 1 \\ q_{1} \cdots q_{k} \mid n}} \lambda_{n}(s) \mu_{n}(s)=\frac{1}{q_{k}^{s}} \sum_{\substack{\theta(n) \geq q_{k} \\ q_{1} \cdots q_{k-1} \mid n}} \lambda_{n}(s)\left(\mu_{n}(s)-\log q_{k}\right) .
$$

Proof. Differentiate (30) and (31) with respect to $s$.

\section{Proof of Theorem 2}

The following estimate for $\Phi(x, y)$, which differs from the one we used in [14], simplifies the proof of Theorem 2 .

Lemma 6. Uniformly, for $x \geq 0, y \geq 2$, we have

$$
\begin{aligned}
\Phi(x, y) & =1_{x \geq 1}+x \prod_{p \leq y}\left(1-\frac{1}{p}\right)+\frac{x}{\log y}\left\{w(u)-e^{-\gamma}-\left.\frac{y}{x}\right|_{x \geq y}+O\left(\frac{e^{-u / 3}}{\log y}\right)\right\} \\
& =1_{x \geq 1}+x \prod_{p \leq y}\left(1-\frac{1}{p}\right)+O\left(\frac{x e^{-u / 3}}{\log y}\right),
\end{aligned}
$$

where $u=\frac{\log \max (1, x)}{\log y}$ and $w(u)$ is Buchstab's function. 
Proof. The second estimate follows from the first, since $w(u)-e^{-\gamma} \ll e^{-u}$ and, if $x \geq y \geq 2$, then $y / x \ll e^{-u / 2}$.

When $x \geq y \geq 2$ and $\log y \geq\left(\log _{2} x\right)^{2}$, the first estimate follows from combining equations (49), (52), (59) and (60) of [11, Sec. III.6], with equation (6) of [11, Sec. III.5], where we estimate the integral in (52) as

$$
\int_{0}^{\infty}\left|w^{\prime}(u-v)\right| y^{-v} d v \ll \int_{0}^{\infty} e^{-(u-v) / 2} y^{-v} d v=\frac{e^{-u / 2}}{\log y-1 / 2} \asymp \frac{e^{-u / 2}}{\log y} .
$$

When $x \geq y \geq 2$ and $\log y<\left(\log _{2} x\right)^{2}$, then $u \gg \sqrt{\log x}$ and the result follows from [11, Thm. III.6.1 and Thm. III.5.1].

When $x<y$, then $\Phi(x, y)=1_{x \geq 1}$ and $w(u)=0$, so that the result follows from Mertens' formula [11, Thm. I.1.11].

Lemma 7. Assume $B(x)=B_{t}(x)$ is the counting function of a set $\mathcal{B}_{t} \subset \mathbb{N}$ that depends on the parameter $t$. Assume

$$
B(x)=x \int_{1}^{\infty} \frac{B(y)}{y^{2} \log y t}\left(e^{-\gamma}-w\left(\frac{\log x / y}{\log y t}\right)\right) d y+R(x) \quad(x \geq 1),
$$

such that the integrals

$$
\alpha_{t}:=e^{-\gamma} \int_{1}^{\infty} \frac{B(y)}{y^{2} \log y t} d y, \quad \beta_{t}:=\frac{-1}{\log t} \int_{1}^{\infty} R(y) \frac{d y}{y^{2}}
$$

converge. Then

$$
B(x)=x \eta_{t} d(v)+O\left\{1+x \beta_{t}(v+1)^{-3.03}+R(x)+I(x)+J(x)\right\},
$$

where $v=\log x / \log t, d(v)$ is given by (11), $\eta_{t}=\alpha_{t}+\beta_{t}$,

$$
I(x)=\frac{x}{\log x t} \int_{x}^{\infty} R(y) \frac{d y}{y^{2}}, \quad J(x)=\frac{x}{(\log x t)^{3.03}} \int_{1}^{x} R(y)(\log y t)^{2.03} \frac{d y}{y^{2}} .
$$

Proof. We follow the second half of the proof of [14, Thm. 1.3]. The only modification needed is the use of the improved estimates

$$
(v+1) d(v)=C+O\left((v+1)^{-2.03}\right), \quad(v \geq 0),
$$

and

$$
(v+1)^{2} d^{\prime}(v)=-C+O\left((v+1)^{-2.03}\right), \quad(v \geq 0)
$$

The estimate (35) is a consequence of [13, Cor. 6], while (36) follows from inserting (35) in the proof of [13, Cor. 5]. In [14], we used slightly weaker estimates for simplicity, with an exponent of -2 instead of -2.03 in the error terms. In the proof of Theorem 2, the improved exponent will save a factor of $\log _{2} x$ (when estimating the contribution from $R_{2}(x)$ to $J(x)$ ).

For $n \in \mathbb{N}$ with prime factorization $n=p_{1} \cdots p_{k}$, where $p_{1} \leq \ldots \leq p_{k}$, define

$$
F(n):=\max _{1 \leq j \leq k} p_{j}^{2} p_{j+1} \cdots p_{k} .
$$

Lemma 8. If $x<\max (m, F(m) / t)$, then $D_{m}(x, t)=0$. 
Proof. Note that $n \in \mathcal{D}_{m}(x, t)$ if and only if $n \leq x, m \mid n$ and $F(n) \leq n t$. Also, $m \mid n$ implies $F(m) \leq F(n)$. Thus, if $D_{m}(x, t) \neq 0$ and $n \in \mathcal{D}_{m}(x, t)$, then $m \leq n \leq x$ and $F(m) \leq F(n) \leq n t \leq x t$, so $x \geq \max (m, F(m) / t)$.

Lemma 9. Let $n \geq 2$ with prime factorization $n=p_{1} \cdots p_{k}, p_{1} \leq \ldots \leq p_{k}$. If $F(n) \leq x$, then $p_{k-j+1} \cdots p_{k} \leq x^{1-2^{-j}}$ for $1 \leq j \leq k$.

Proof. We use induction on $j$. When $j=1$, the claim is that $p_{k} \leq x^{1 / 2}$, which follows from $p_{k}^{2} \leq F(n) \leq x$, for all $k \geq 1$. Assume now that the claim is correct for some $j \in \mathbb{N}$ and all $k \geq j$. Let $k \geq j+1$. We have $p_{k-j}^{2} p_{k-j+1} \cdots p_{k} \leq F(n) \leq x$. Thus, if $p_{k-j} p_{k-j+1} \cdots p_{k} \geq x^{1-2^{-j-1}}$, then $p_{k-j} \leq x^{2^{-j-1}}$. By the inductive hypothesis,

$$
p_{k-j}\left(p_{k-j+1} \cdots p_{k}\right) \leq x^{2^{-j-1}} x^{1-2^{-j}}=x^{1-2^{-j-1}},
$$

for all $k \geq j+1$.

Lemma 10. Let $k \geq 0$ be fixed. For $m \geq 1$ with $\Omega(m)=k, t \geq 2$ and $x \geq \max (m, F(m) / t)$, we have

$$
\frac{x \log t \log 2 m}{m \log x t \log 2 x} \gg_{k} 1
$$

Proof. This is obvious if $m=1$ or if $t \geq x^{2^{-k}}$. If $m \geq 2$ and $t<x^{2^{-k}}$, then $F(m) \leq x t$ and Lemma 9 imply $m \leq(x t)^{1-2^{-k}}<x^{1-4^{-k}} \ll_{k} x /(\log x t)^{2}$, from which the claim follows.

Proof of Theorem 2. In the remainder of this paper, we write $D_{q}(x)$ for $D_{q}(x, t)$ and $D(x)$ for $D(x, t)$. We will show by induction on $k \geq 0$ that, for $k=\Omega(q)$, the estimates (12), (13) and (14) hold and that, for primes $r$ with $r \geq P^{+}(q)$, we have

$$
\sum_{\substack{n \in \mathcal{D}_{q} \\ n \geq r / t}} \Phi(x / r n, n t)-\frac{x}{r} \sum_{\substack{n \in \mathcal{D}_{q} \\ n \geq r / t}} \lambda_{n} \ll 1+\frac{x \log t \log q r \log q r t}{q r \log ^{3} x t}=: R_{2}(x, q r),
$$

for $x \geq \max (q r, F(q r) / t)$. Note that (13) and (35) imply

$$
D_{q}(x) \ll_{k} x d(v) \eta_{q, t} \asymp_{k} \frac{x \log t}{q \log x t}, \quad(x \geq 1, q \geq 1, t \geq 2),
$$

since $D_{q}(x)=0$ if $q>x$.

When $k=0, q=1,(12)$ is [14, Eq. (13)], (13) is [14, Thm. 1.3] and (14) is [14, Eq. (6)]. To show (37) for $k=0$ and $q=1$, assume that $r$ is prime 
and $x \geq \max \left(r, r^{2} / t\right)$. Equations (27) and (30) show that

$$
\begin{aligned}
& \sum_{\substack{n \in \mathcal{D} \\
n \geq r / t}} \Phi(x / r n, n t)-\frac{x}{r} \sum_{\substack{n \in \mathcal{D} \\
n \geq r / t}} \lambda_{n} \\
& =-\{x / r\}-\sum_{\substack{n \in \mathcal{D} \\
n<r / t}} \Phi(x / r n, n t)+\frac{x}{r} \sum_{\substack{n \in \mathcal{D} \\
n<r / t}} \lambda_{n} \\
& \ll 1+D(r / t)+\sum_{\substack{n \in \mathcal{D} \\
n<r / t}} \frac{x}{r n \log n t} \exp \left(-\frac{\log x t / r}{3 \log n t}\right),
\end{aligned}
$$

by the second estimate in Lemma 6 . We have $D(r / t) \ll r \log t /(t \log r)$ by Lemma 1 , so $D(r / t) \ll R_{2}(x, r)$ follows from

$$
\frac{r}{\log ^{2} r \log r t} \ll \frac{x t / r}{\log ^{3} x t} \asymp \frac{x t / r}{\log ^{3}(x t / r)},
$$

since $x t / r \geq \sqrt{x t}$. This holds because $r \leq x t / r$. Finally, the last sum in (39) is $\ll R_{2}(x, r)$ by Lemma 1 . Thus, (37) holds for $k=0$.

For the inductive step, assume that (13) (and hence (38)) and (14) hold for $q \in \mathbb{N}$ with $\Omega(q)=k$ for some $k \geq 0$. If $k \geq 1$, assume that (37) holds for $\Omega(q)=k-1$. Let $r$ be a prime with $r \geq P^{+}(q)$ and write $m=q r$. We note that in the remainder of this proof, all implied constants in the $\ll$ and big-O notation may depend on $k$.

We estimate the first sum in (28) with Lemma 6 and apply (31) to get

$$
\begin{aligned}
D_{m}(x) & +\sum_{n \in \mathcal{D}_{m}} \frac{x}{n \log n t}\left\{w\left(\frac{\log x / n}{\log n t}\right)-e^{-\gamma}-\left.\frac{n^{2} t}{x}\right|_{n^{2} \leq \frac{x}{t}}+O\left(\frac{e^{-\frac{\log x / n}{\log n t}}}{\log n t}\right)\right\} \\
& =\sum_{\substack{n \in \mathcal{D}_{q} \\
n \geq r / t}} \Phi(x / r n, n t)-\frac{x}{r} \sum_{\substack{n \in \mathcal{D}_{q} \\
n \geq r / t}} \lambda_{n} .
\end{aligned}
$$

The contribution from the last two terms in the first sum in (40) is

$$
\ll \tilde{R}_{1}(x):=\frac{x \log m t}{m(\log x t)^{2}},
$$

by Lemma 1. In the second application of this argument we will be able to replace $\tilde{R}_{1}(x)$ by the smaller

$$
R_{1}(x):=\frac{x \log t}{m(\log x t)^{2}} .
$$

The error from applying Abel summation to the remaining terms of the first sum in $(40)$ is also $\ll \tilde{R}_{1}(x)$, since $w(u)-e^{-\gamma} \ll e^{-u}$ and $w^{\prime}(u) \ll e^{-u}$. 
Thus,

$$
\begin{aligned}
D_{m}(x)= & x \int_{1}^{\infty} \frac{D_{m}(y)}{y^{2} \log y t}\left(e^{-\gamma}-w\left(\frac{\log x / y}{\log y t}\right)\right) d y+O\left(\tilde{R}_{1}(x)\right) \\
& +\sum_{\substack{n \in \mathcal{D}_{q} \\
n \geq r / t}} \Phi(x / r n, n t)-\frac{x}{r} \sum_{\substack{n \in \mathcal{D}_{q} \\
n \geq r / t}} \lambda_{n} .
\end{aligned}
$$

If $x / r<\max (q, F(q) / t, r / t)$, that is $x<\max (m, F(m) / t)$, then Lemma 8 shows that the first sum in (41) vanishes, while the second sum is

$$
\ll \frac{x}{m} \min \left(1, \frac{\log t}{\log r}\right) \ll \frac{x}{m} \min \left(1, \frac{\log t}{\log m}\right),
$$

by the inductive hypothesis (38) and since $\log r \leq \log m \leq \log r^{k+1} \ll_{k} \log r$. Define

$$
R_{2}(x):= \begin{cases}\frac{x}{m} \min \left(1, \frac{\log t}{\log m}\right) & \text { if } x<\max (m, F(m) / t) \\ 1+\frac{x \log t \log m \log m t}{m \log ^{3} x t} & \text { if } x \geq \max (m, F(m) / t) .\end{cases}
$$

Thus,

$$
D_{m}(x)=x \int_{1}^{\infty} \frac{D_{m}(y)}{y^{2} \log y t}\left(e^{-\gamma}-w\left(\frac{\log x / y}{\log y t}\right)\right) d y+R(x)
$$

holds with $R(x) \ll \tilde{R}_{1}(x)+R_{2}(x)$ when $x<\max (m, F(m) / t)$.

Assume now that $x \geq \max (m, F(m) / t)$. If $k=0$ and $q=1$, we have already shown that the last row of $(41)$ is $\ll R_{2}(x)$. If $k \geq 1$, write $q=u v$ where $v=P^{+}(q)$. Equations (28) and (31) show that the last row of (41) equals

$$
\begin{aligned}
& =\sum_{\substack{n \in \mathcal{D}_{u} \\
n \geq v / t}} \Phi(x / n r v, n t)-\frac{x}{r v} \sum_{\substack{n \in \mathcal{D}_{u} \\
n \geq v / t}} \lambda_{n}-\sum_{\substack{n \in \mathcal{D}_{q} \\
n<r / t}} \Phi(x / n r, n t)+\frac{x}{r} \sum_{\substack{n \in \mathcal{D}_{q} \\
n<r / t}} \lambda_{n} \\
& =S-T-(U-V),
\end{aligned}
$$

say. Now $x \geq \max (m, F(m) / t)$ implies $x / r \geq \max (q, F(q) / t, r / t)$. The inductive hypothesis (37) yields

$$
S-T \ll 1+\frac{(x / r) \log t \log q \log q t}{q \log ^{3}(x t / r)} \ll 1+\frac{x \log t \log m \log m t}{m \log ^{3} x t}=R_{2}(x),
$$

since $x t / r \geq \sqrt{x t}$. The second estimate in Lemma 6 and (38) show that

$$
\begin{aligned}
U-V & \ll D_{q}(r / t)+\sum_{\substack{n \in \mathcal{D}_{q} \\
n<r / t}} \frac{x}{n r \log n t} \exp \left(-\frac{\log \frac{x}{n r}}{3 \log n t}\right) \\
& \ll \frac{r^{2} \log t}{m t \log r}+R_{2}(x) \ll R_{2}(x),
\end{aligned}
$$


where the last assertion is implied by

$$
\frac{r}{\log r \log m \log m t} \ll \frac{x t / r}{\log ^{3}(x t / r)},
$$

which holds because $m t>m \geq r$ and $r \leq x t / r$. As the last row of (41) is $\ll R_{2}(x)$, we have established that (37) holds for $\Omega(q) \leq k$ and that (42) holds with $R(x) \ll \tilde{R}_{1}(x)+R_{2}(x)$.

We need to estimate $I(x)$ and $J(x)$ from Lemma 7 . For this purpose we may assume that $x \geq \max (m, F(m) / t)$. If not, Lemma 8 shows that $D_{m}(x)=0$, so that (12) holds since the main term in (12) is absorbed by the error terms. We find that $I(x)+J(x) \ll \tilde{R}_{1}(x)+R_{2}(x)$ and $\beta_{t} \ll \frac{\log m}{m \log t}$ so that $x \beta_{t}(v+1)^{-3} \ll R_{2}(x)$.

The conclusion of Lemma 7 is that

$$
D_{m}(x)=x \eta_{m, t} d(v)+O\left(\tilde{R}_{1}(x)+R_{2}(x)\right) .
$$

The lower bound in Lemma 1 yields $\eta_{m, t} \gg m^{-1}$. Lemma 11 shows that $\eta_{m, t} \ll_{k} m^{-1}$. Together with (43), Lemmas 8 and 10, this implies

$$
D_{m}(x) \ll_{k} \frac{x \log t}{m \log x t}, \quad(x \geq 1, t \geq 2, \Omega(m) \leq k+1) .
$$

Running through this proof a second time with this upper bound replacing the one in Lemma 1 , we can replace $\tilde{R}_{1}(x)$ by $R_{1}(x)$ to obtain

$$
D_{m}(x)=x \eta_{m, t} d(v)+O\left(R_{1}(x)+R_{2}(x)\right),
$$

where $1 / m \ll \eta_{m, t} \ll_{k} 1 / m$. This shows that (12) and (14) hold with $q$ replaced by $m=q r$.

To see that (12) implies (13), note that the term $O(1)$ is acceptable by Lemma 10, provided $x \geq \max (m, F(m) / t)$. If $x<\max (m, F(m) / t)$, then $D_{m}(x)=0$ and $x<F(m) \leq m^{2}$, so (13) (with $q$ replaced by $m$ ) follows from $\log x \leq 2 \log m$. This completes the proof of Theorem 2 .

Lemma 11. Assume that (43) holds for $m$ with $\Omega(m) \leq k+1$, and that $\eta_{q, t} \ll_{k} q^{-1}$ for $\Omega(q) \leq k$. Then $\eta_{m, t} \ll_{k} m^{-1}$ for $\Omega(m)=k+1$.

Proof. Let $m=q r$ where $r$ is prime and $r \geq P^{+}(q)$. Equation (34) yields

$$
\sum_{\substack{n \geq 1 \\ q r \mid n}} \lambda_{n}(s) \mu_{n}(s)=\frac{1}{r^{s}} \sum_{\substack{n \geq r / t \\ q \mid n}} \lambda_{n}(s)\left(\mu_{n}(s)-\log r\right) .
$$

As in [16], we let $s=1+1 / \log ^{2} N$ and split the sum on the left according to $n \leq N$ and $n>N$. As $N \rightarrow \infty$, the contribution from $n \leq N$ converges to (see [16, Lemma 3]) $\sum_{q r \mid n} \lambda_{n} \mu_{n}$, while the contribution from $n>N$ converges to (see [16, Lemma 4]) $-c_{q r}\left(1-e^{-\gamma}\right)$, by (43), where $\eta_{q r, t}=$ $C c_{q r} \log t$. Applying the same reasoning to the sum on the right-hand side, 
we get

$$
\sum_{\substack{n \geq 1 \\ q>\mid n}} \lambda_{n} \mu_{n}-c_{q r}\left(1-e^{-\gamma}\right)=\frac{1}{r}\left(\sum_{\substack{n \geq r / t \\ q \mid n}} \lambda_{n}\left(\mu_{n}-\log r\right)-c_{q}\left(1-e^{-\gamma}\right)\right) .
$$

Since $\mu_{n} \ll \log t$, we obtain

$$
r c_{q r} \ll S \log t+S \log r+c_{q}, \quad S:=r \sum_{\substack{n \geq 1 \\ q r \mid n}} \lambda_{n}=\sum_{\substack{n \geq r / t \\ q \mid n}} \lambda_{n},
$$

by (31). Now $\eta_{q, t} \ll q^{-1}$ and (44) holds with $q$ in place of $m$. Thus, $c_{q} \ll q^{-1} \log t$ and $S=\sum_{\substack{n \geq r / t \\ q \mid n}} \lambda_{n} \ll q^{-1} \min (1, \log t / \log r)$. This shows that $r c_{q r} \ll q^{-1} \log t$, which is the desired result.

\section{Proof of Theorem 3}

Proof of (18), (19) and (20). The estimate (18) is [16, Cor. 3]. Equation (34), with $k=1$ and $q_{1}=q$ prime, is

$$
\sum_{\substack{n \geq 1 \\ q \backslash n}} \lambda_{n}(s) \mu_{n}(s)=\frac{1}{q^{s}} \sum_{n \geq q / t} \lambda_{n}(s)\left(\mu_{n}(s)-\log q\right) .
$$

As in [16], we let $s=1+1 / \log ^{2} N$ and split the sum on the left according to $n \leq N$ and $n>N$. As $N \rightarrow \infty$, the contribution from $n \leq N$ converges to (see [16, Lemma 3]) $\sum_{\substack{n \geq 1 \\ q \mid n}} \lambda_{n} \mu_{n}$, while the contribution from $n>N$ converges to (see [16, Lemma 4]) $-c_{q}\left(1-e^{-\gamma}\right)$, by Corollary 6. Applying the same reasoning to the right-hand side of (45), we get

$$
\sum_{\substack{n \geq 1 \\ q \mid n}} \lambda_{n} \mu_{n}-c_{q}\left(1-e^{-\gamma}\right)=\frac{1}{q}\left(\sum_{n \geq q / t} \lambda_{n}\left(\mu_{n}-\log q\right)-c_{\theta}\left(1-e^{-\gamma}\right)\right) .
$$

With the estimate [16, Lemma 13]

$$
\mu_{n}=\log t-\gamma+O\left(e^{-\sqrt{\log n t}}\right)
$$

we obtain

$$
q c_{q}\left(1-e^{-\gamma}\right)=S \log q+c_{\theta}\left(1-e^{-\gamma}\right)+O\left(S e^{-\sqrt{\log \max (q, t)}}\right),
$$

where

$$
S:=\sum_{n \geq q / t} \lambda_{n}=q \sum_{\substack{n \geq 1 \\ q \mid n}} \lambda_{n}
$$

by (31). If $q \leq t$, then $S=1$ by (30), so that (20) follows from (47).

In general, we have

$$
S=\frac{e^{-\gamma} c_{\theta}}{\log q}\left(1+O\left(\frac{1}{\log q}+\frac{\log ^{2} t}{\log ^{2} q}\right)\right), \quad(q \geq 2, t \geq 2) .
$$


If $q \leq t$, this holds because $S=1$ and $c_{\theta} \asymp \log t$. If $q \geq t$, (48) follows from applying Abel summation to $S=\sum_{n \geq q / t} \lambda_{n}$, together with Mertens' formula and Corollary 6 (with $q=1$ ). Combining (48) with (47) proves (19).

Proof of (21), (22) and (23). Equation (34), with $k=2$ and $q_{1}=p \leq q_{2}=$ $q$ is

$$
\sum_{\substack{n \geq 1 \\ p q \mid n}} \lambda_{n}(s) \mu_{n}(s)=\frac{1}{q^{s}} \sum_{\substack{n \geq q / t \\ p \mid n}} \lambda_{n}(s)\left(\mu_{n}(s)-\log q\right) .
$$

As in [16], we let $s=1+1 / \log ^{2} N$ and split the sum on the left according to $n \leq N$ and $n>N$. As $N \rightarrow \infty$, the contribution from $n \leq N$ converges to (see [16, Lemma 3]) $\sum_{p q \mid n} \lambda_{n} \mu_{n}$, while the contribution from $n>N$ converges to (see [16, Lemma 4]) $-c_{p q}\left(1-e^{-\gamma}\right)$, by Corollary 6. Applying the same reasoning to the right-hand side of (49), we get

$$
\sum_{\substack{n \geq 1 \\ p q \mid n}} \lambda_{n} \mu_{n}-c_{p q}\left(1-e^{-\gamma}\right)=\frac{1}{q}\left(\sum_{\substack{n \geq q / t \\ p \mid n}} \lambda_{n}\left(\mu_{n}-\log q\right)-c_{p}\left(1-e^{-\gamma}\right)\right) .
$$

We estimate $\mu_{n}$ with (46) to obtain

$$
q c_{p q}\left(1-e^{-\gamma}\right)=T \log q+c_{p}\left(1-e^{-\gamma}\right)+O(T \exp (-\sqrt{\log \max (p t, q)})),
$$

where

$$
T:=\sum_{\substack{n \geq q / t \\ p \mid n}} \lambda_{n}=q \sum_{\substack{n \geq 1 \\ p q \mid n}} \lambda_{n}
$$

by $(31)$.

If $p \leq q \leq t$, then

$$
T=\sum_{\substack{n \geq 1 \\ p \mid n}} \lambda_{n}=\frac{1}{p} \sum_{n \geq p / t} \lambda_{n}=\frac{1}{p} \sum_{n \geq 1} \lambda_{n}=\frac{1}{p},
$$

by (31) and (30). The estimate (23) now follows from (50) with $T=1 / p$ and $c_{p}$ estimated by (20).

In general, we have

$$
T=\frac{e^{-\gamma} c_{p}}{\log q}\left(1+O\left(\frac{1}{\log q}+\frac{\log ^{2} p t}{\log ^{2} q}\right)\right), \quad(q \geq p \geq 2, t \geq 2) .
$$

If $q<p t$, this is implied by $T \ll c_{p} / \log q$, which follows from (17) (with $q$ replaced by $p$ ). If $q \geq p t$, we estimate $\lambda_{n}$ with Mertens' formula and use Abel summation and the estimate (15). The contribution from the first two error terms in (15) is clearly acceptable, while the term $O(1)$ contributes

$$
\ll \int_{q / t}^{\infty} \frac{d y}{y^{2} \log y t} \leq \frac{t}{q \log q} \asymp \frac{c_{p}}{\log q} \cdot \frac{p t}{q \log t} \ll \frac{c_{p}}{\log q} \cdot \frac{\log ^{2} p t}{\log ^{2} q} .
$$

Now substitute (51) into (50) and estimate $c_{p}$ with (19) to get (21), and with (20) to get (22). 


\section{Proof of Theorem 1}

Proof of (1). We have

$$
\sum_{n \in \mathcal{D}(x)} \omega(n)=\sum_{p \leq x} D_{p}(x)=x d(v) \sum_{p \leq x} \eta_{p, t}\left(1+O\left(\frac{\log p}{\log x}\right)\right),
$$

by (13). The contribution from the error term is $\ll x d(v) \asymp D(x)$, since $\eta_{p, t} \ll 1 / p$ by (14). Now $\eta_{p, t} C \log t=c_{p}$ and $\eta_{1, t} C \log t=c_{\theta}$, by Corollary 6. With (18), (19) and (20), we find that

$$
\sum_{p \leq x} \eta_{p, t}=\frac{1}{C \log t} \sum_{p \leq x} c_{p}=\frac{c_{\theta}}{C \log t}(E(x, t)+O(1))=\eta_{1, t}(E(x, t)+O(1)) .
$$

The result now follows from (13) with $q=1$, that is $D(x)=x d(v) \eta_{1, t}(1+$ $O(1 / \log x))$.

To see that (1) remains valid when $\omega$ is replaced by $\Omega$, note that

$$
\sum_{n \in \mathcal{D}(x)}(\Omega(n)-\omega(n))=\sum_{n \in \mathcal{D}(x)} \sum_{k \geq 2} \sum_{p^{k} \mid n} 1=\sum_{p \leq x} \sum_{k \geq 2} D_{p^{k}}(x) \ll \frac{x \log t}{\log x t} \ll D(x),
$$

by Lemma 1 .

Proof of (2). We have

$$
\sum_{n \in \mathcal{D}(x)} \omega(n)^{2}=\sum_{p, q \leq x} D_{p q}(x)+O\left(\sum_{p \leq x} D_{p}(x)\right),
$$

where $p, q$ run over primes. The last term is $\ll D(x) \log _{2} x$, by (1). Thus, (13) yields

$$
\sum_{n \in \mathcal{D}(x)} \omega(n)^{2}=O\left(D(x) \log _{2} x\right)+x d(v) \sum_{p, q \leq x} \eta_{p q, t}\left(1+O\left(\frac{\log p q}{\log x}\right)\right) .
$$

Since $\eta_{p q, t} \ll 1 / p q$, the contribution from the error term is $\ll x d(v) \log _{2} x \ll$ $D(x) \log _{2} x$. With (21), (22) and (23), we find that

$$
\begin{aligned}
\sum_{p, q \leq x} \eta_{p q, t}=\frac{1}{C \log t} \sum_{p, q \leq x} c_{p q} & =\frac{c_{\theta}}{C \log t}\left(E(x, t)^{2}+O\left(\log _{2} x\right)\right) \\
& =\eta_{1, t}\left(E(x, t)^{2}+O\left(\log _{2} x\right)\right) .
\end{aligned}
$$

Combining this with (1) and (13) (with $q=1$ ), we get

$$
\sum_{n \in \mathcal{D}(x)}(\omega(n)-E(x, t))^{2} \ll D(x) \log _{2} x
$$

which implies (2). This estimate remains valid if $\omega(n)$ is replaced by $\Omega(n)$, since

$$
\sum_{n \in \mathcal{D}(x)}(\Omega(n)-\omega(n))^{2} \leq \sum_{p \neq q \leq x} \sum_{k, j \geq 2} D_{p^{k} q^{j}}(x)+\sum_{p \leq x} \sum_{k \geq 2} 2 k D_{p^{k}}(x) \ll D(x),
$$

by Lemma 1 . 


\section{ACKNOWLEDGMENTS}

The author thanks Eric Saias and the anonymous referee for several very helpful suggestions.

\section{REFERENCES}

[1] R. R. Hall and G. Tenenbaum, Divisors, Cambridge University Press, 1988.

[2] M. Margenstern, Les nombres pratiques: théorie, observations et conjectures, J. Number Theory 37 (1991), 1-36.

[3] J.-L. Nicolas, Sur la distribution des nombres entiers ayant une quantité fixée de facteurs premiers, Acta Arith. 44 (1984), 191-200.

[4] C. Pomerance, L. Thompson, A. Weingartner, On integers $n$ for which $X^{n}-1$ has a divisor of every degree, Acta Arith. 175 (2016), no. 3, 225-243.

[5] C. Pomerance and A. Weingartner, On primes and practical numbers, arXiv: 2007.11062

[6] E. Saias, Entiers à diviseurs denses 1, J. Number Theory 62 (1997), 163-191.

[7] W. Sierpiński, Sur une propriété des nombres naturels, Ann. Mat. Pura Appl. (4) 39 (1955), 69-74.

[8] A. K. Srinivasan, Practical numbers, Current Sci. 17 (1948), 179-180.

[9] B. M. Stewart, Sums of distinct divisors, Amer. J. Math. 76 (1954), 779-785.

[10] G. Tenenbaum, Sur un problème de crible et ses applications, Ann. Sci. École Norm. Sup. (4) 19 (1986), 1-30.

[11] G. Tenenbaum, Introduction to Analytic and Probabilistic Number Theory, Cambridge Studies in Advanced Mathematics, Vol. 46, Cambridge Univ. Press, Cambridge, 1995.

[12] L. Thompson, Polynomials with divisors of every degree, J. Number Theory 132 (2012), 1038-1053.

[13] A. Weingartner, Integers with dense divisors 3, J. Number Theory 142 (2014), 211222 .

[14] A. Weingartner, Practical numbers and the distribution of divisors, Q. J. Math. 66 (2015), no. 2, 743-758.

[15] A. Weingartner, A sieve problem and its application, Mathematika 63 (2017), no. 1, $213-229$.

[16] A. Weingartner, On the constant factor in several related asymptotic estimates, Math. Comp. 88 (2019), no. 318, 1883-1902.

[17] A. Weingartner, The mean number of divisors for rough, dense and practical numbers, preprint arXiv:2104.07137.

Department of Mathematics, 351 West University Boulevard, Southern Utah University, Cedar City, Utah 84720, USA

Email address: weingartner@suu.edu 